Anterior Staphyloma of The Cornea

Tuberculosis death rate over last 21 years per 1,000,000

\begin{tabular}{r|r|r|r}
\hline Year & Respiratory & Other Forms & Both \\
\hline $1908-1912$ & 855 & 182 & 1037 \\
1921 & 667 & 137 & 804 \\
1922 & 565 & 120 & 685 \\
1923 & 620 & 123 & 743 \\
1924 & 585 & 127 & 712 \\
1925 & 561 & 97 & 658 \\
1926 & 545 & 84 & 629 \\
1927 & 546 & 100 & 646 \\
1928 & 581 & 82 & 663 \\
1929 & 496 & 92 & 588 \\
1930 & 493 & 97 & 595 \\
1931 & 481 & 68 & 549 \\
1932 & 450 & 76 & 526 \\
1933 & 405 & 66 & 471 \\
1934 & 411 & 74 & 485 \\
1935 & 414 & 64 & 478 \\
1936 & 412 & 59 & 471 \\
1937 & 384 & 51 & 435 \\
1938 & - & - & - \\
\hline
\end{tabular}

\title{
RESECTION OF ANTERIOR STAPHYLOMA OF THE CORNEA BY MEANS OF GRADUAL INCISIONS
}

B Y

\author{
N. I. SHIMKIN \\ HAIFA, PALESTINE
}

ANTERIOR staphyloma of the cornea in European countries is more rare than in sub-tropical ones, such as Palestine.

Epidemics of acute conjunctivitis, especially during the dry and hot seasons of the year, aided by the exceedingly widespread trachoma, lead to frequent ulceration of the cornea which is one of the main causes of blindness among the population of Palestine. (Strathearn, Shimkin, Ticho.) 
Therefore, corneal staphyloma in various forms and dimensions is often met with in this country, although less than formerly, thanks to the Palestinian Government having built good roads and convenient ways of communication which enable the patient quickly to apply to an eye-surgeon for special treatment.

The Arabic peasant population (Fellaheen) consult an oculist about an anterior staphyloma of the cornea either when it has reached such a size that it is not covered by the lids, or when the apex of such a staphyloma, exposed to the influence of the wind and dust, has become ulcerated and causes the patient insupportable pain.

Usually these patients ask the eye surgeon whether he can keep the eye-ball intact. The offer to enucleate or eviscerate the eyeball is definitely rejected by these patients. Their opinion is that a man possessing an eye with an anterior staphyloma is still " a man like the others," while a man without an eye-ball is a cripple. Besides, many peasants point out with a reason that it is difficult to work in a field with an artificial eye, especially during the hot time of the year, and the artificial eye may break, necessitating further expenses to replace it.

Along with the cultural progress and the economic improvement of the situation of the Arabic peasantry in Palestine, this prejudice against enucleation or evisceration of the eye, is gradually disappearing. However, in countries infected with trachoma and suffering also from different forms of acute conjunctivitis, the anterior staphyloma of the cornea, needing operation, will still be met with for a long time.

All operations for anterior staphyloma of the cornea, described in handbooks of eye-surgery, differ from each other only in the method of inserting sutures in the margins of the wound after the resection of the anterior staphyloma. But in all these methods the resected part of the corneal staphyloma is removed in one stroke, and the borders of the ensuing large and widely open wound are drawn together by means of sutures, inserted beforehand, and lying either under the conjunctiva or in the tissue of wound margin.

The most frequent complication following this method of operation is loss of vitreous. The larger the segment of the resected anterior staphyloma, the bigger is the opening of the wound, and the greater is the danger of losing vitreous. This danger is increased by the fact that the vitreous humour in large and old staphylomata is usually strongly liquefied, and flows out like water from the distended eye ball. Certainly a small loss of vitreous is restored, but sometimes its loss is so great that the operation for the staphyloma has to be followed by an evisceration 
of the eye-ball, i.e. by the very operation which the patient has not consented to.

In order to avoid this complication, namely, the great loss of vitreous humour, I suggest the following method of operation on anterior corneal staphyloma. This method was applied in 31 cases on out-patients of my clinic during the 18 years of practising eyesurgery in Palestine.

The essence of this method consists in this, that the anterior staphyloma of the cornea of whatever size, is resected not at once but by gradual incisions, each $1 \frac{1}{2}-2 \mathrm{~mm}$. wide. The margins of each incision are immediately drawn together by sutures. The execution of the operation is so simple that it may be performed on out-patients without the aid of an assistant.

Instruments required: An eye speculum, fixation forceps, a sharp-pointed scalpel, blunt-pointed eye scissors, 7-8 silk threads with curved needles, a needle-holder, a 2 c.c. syringe with medium size needle.

Anaesthesia.-Retrobulbar injection of 2 c.c. novocaine 2 per cent. + adrenalin, akinesia of the lids by means of the same solution and a subconjunctival injection of 1 c.c. of the same solution in the region of the ciliary body; instillation of drops of 5 per cent. cocaine three times, 2 drops every 3 minutes. Usually by the end of the instillation a total anaesthesia of the eye-ball and a total akinesia of the lids have occurred.

\section{The Operation}

Step I.-(a) The lids are widely opened by means of the eye speculum. If adhesions of the conjunctiva of the lid with the eye-ball are present, they are cut with scissors and then the eyeball is quite free from any tension, and does not suffer any pressure from the eye speculum.

(b) Boundaries of the part of staphyloma to be resected, are outlined by means of the sharp point of the scalpel. This part has usually an ovoid form. The long axis of this ovoid lies horizontal, the outer and inner margins of the ovoid must be $\frac{1}{4} \mathrm{~mm}$. distant from the neighbouring sclera (Fig. I).

Step II.-By means of the sharp point of the scalpel, the outer end of the ovoid is cut through on the lower and upper margins, for a distance of $1 \frac{1}{2} \mathrm{~mm}$. from its outer end. Through this wound the liquid of the anterior chamber slowly flows out. The end of the ovoid, cut through, is caught up by means of fixation forceps, and in the lips of the wound a suture is inserted, which is immediately tied by means of a surgical knot. (Fig. II).

Step III.- The resected part of the staphyloma is lifted upwards without any tension and the incision is lengthened $1 \frac{1}{2}-2 \mathrm{~mm}$. along 
the outlined boundary; the lips of the wound are immediately sewn together by means of a suture which is tied with a surgical knot. (Figs. II and III).

Step IV, V, VI, VII.-These steps are a repetition of Step III, i.e., the resected part of the staphyloma is lifted upwards with fixation forceps without any tension, and each time the incision is lengthened $1 \frac{1}{2}-2 \mathrm{~mm}$. inwards, both above and below.

After each incision, a suture is immediately inserted in the lips of the wound, and tied by means of a surgical knot. (Figs. III and IV).

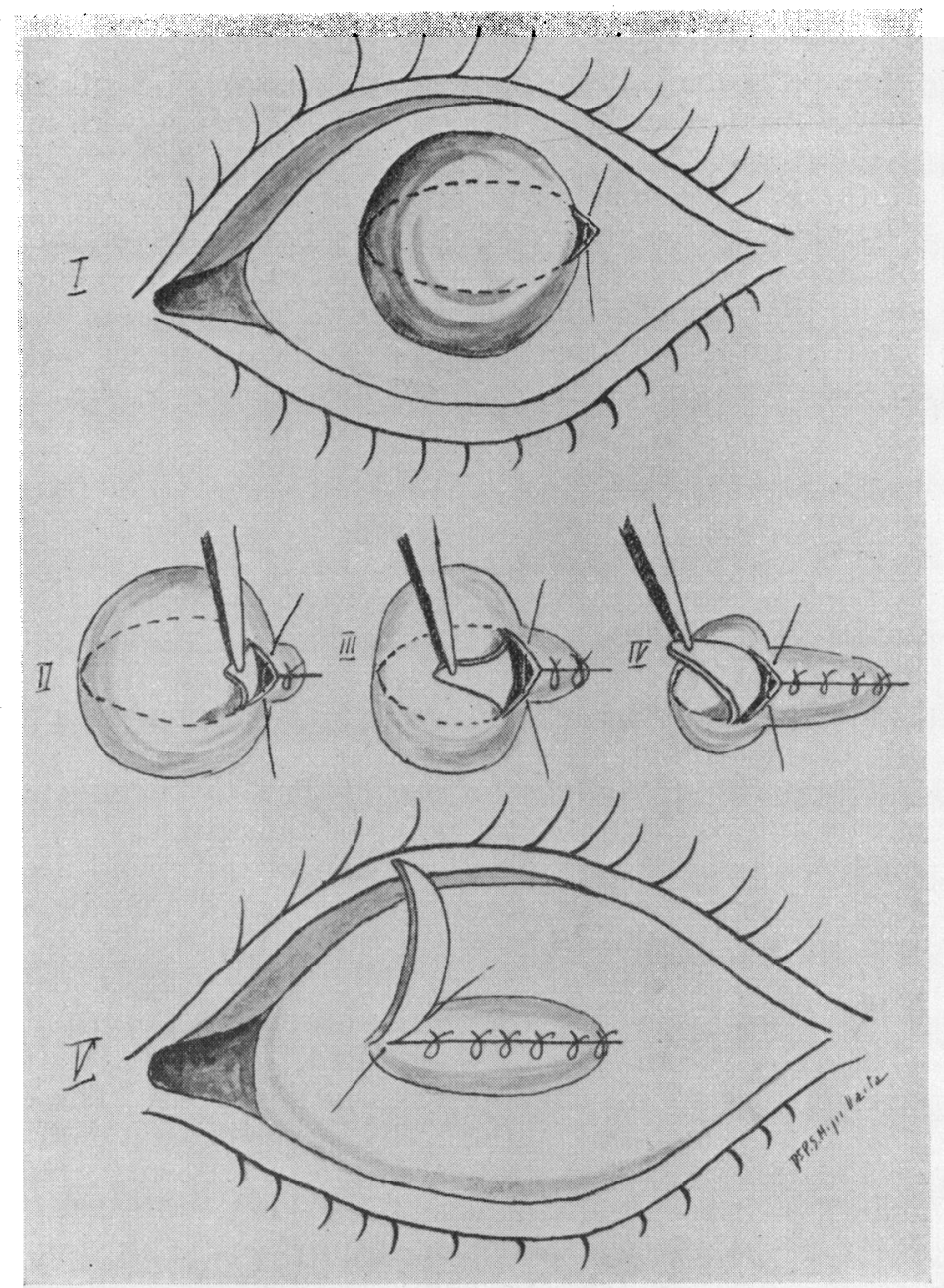


Step VIII.-After the 6 sutures have been inserted, the staphyloma, now resected almost completely, is drawn with forceps upwards, and the last suture is inserted through the lips of the remaining wound at the extreme inner border of the ovoid; then the resected staphyloma is cut away altogether, and the suture is tied with a surgical knot. (Fig. V).

The ends of the threads are cut away not too short with scissors, and the sutures are painted over with iodine; 5 per cent., xeroform ointment is put in and both eyes are bandaged for 48 hours.

Usually, 7 sutures are inserted but if the anterior staphyloma is very big, 8 sutures are needed.

Post-operative Treatment.-Immediately after the operation, the patient is sent home. (All the cases operated on were on outpatients.)

The first bandage is changed after 48 hours. The eye is washed with 2 per cent. solution boric acid, 5 per cent. cocaine is instilled, and 5 per cent. xeroform ointment put in; a bandage is applied to both eyes for another 48 hours. The sutures are removed on the 5th day. Before removing the sutures, a thorough anaesthesia of the eye should be made by means of instillation 5 per cent. cocaine. If the anaesthesia of the eye is not sufficient the patient may suddenly jerk his head backwards while the sutures are being caught by the forceps, the suture may tear the cornea, and the wound margins in that place may separate. This complication does not lead to serious consequences but the postoperative treatment is lengthened by several days until the lips of the wound grow together again.

After the sutures have been removed, only a bandage is applied to the eye operated on. Usually, after 7-8 days, the patient is cured. The eye-ball retains its usual form, and is well covered by the lids; the upper lid does not fall inside and backwards, as occurs after an enucleation of the eye-ball.

For a successful issue of the operation, it is essential that the curved needles should be sharp-pointed, in order to pass easily through the thickness of the cornea, without effecting any tension on the eye-ball walls; the needle-holder should open easily so that no concussion of the eye-ball walls is made while the lock is opened.

In the operation for conical staphyloma of the cornea, where the walls are very thin, it is advisable when sewing the lips of the wound, that the needle passes through all of the thinned wall, $2-1 \frac{1}{2} \mathrm{~mm}$. away from its border. When excising staphyloma with thick sclerotic walls, the needle is made to pass in the thickness of the wall $1 \mathrm{~mm}$. away from its border.

The insertion of sutures is made easier if, when lifting up the resected part of the staphyloma by means of the forceps, the 
surgeon simultaneously slightly lifts up the lips of the wound margins also. This enables the surgeon to see at what distance from the margin of the lips of the wound the needle enters, and also, whether the stitch lies in the thickness of tissue, or passes through it. The surgeon also sees how thickly, in certain cases of staphyloma, the back (inside) surface of its wall is covered by the distended and atrophied iris, and he may easily avoid wounding the still remaining part of the iris. The surgeon may still easily avoid wounding the lens which lies much lower than the wound margins, which are being sewn together. But the major convenience of this operation of resection of anterior staphyloma by means of incision consists in that, that the area of the open wound in the eye-ball is always very small; the wound margins are at a distance of $1 \frac{1}{2}-2 \mathrm{~mm}$. from each other; when bringing together the lips of the wound by means of a suture, the walls of the eye-ball do not suffer any tension and therefore the danger of losing the vitreous is reduced to a minimum.

The writer desires to point out that he does not remove the lens as is required with other methods of staphyloma operations, and has never seen any bad consequences. On the contrary, he considers that the lens in a capsule strengthened by the ligament of Zinn acts as a closed diaphragm, preventing the vitreous humour from flowing out. Besides, in anterior staphyloma, which has developed in childhood, the lens is usually missing, and therefore there is nothing to remove.

\section{Summary}

The author suggests performing resection of anterior staphyloma of the cornea by means of gradual incisions $1 \frac{1}{2}-2 \mathrm{~mm}$. wide each. The lips of the open wound of eye-ball are immediately sewn together after each incision. Thanks to this method, the danger of loss of vitreous is minimized. By this method of staphyloma resection the lens is not removed. The author has operated by this method on 31 cases of anterior staphyloma on his outpatients during 18 years, with quite satisfactory results. In the table given herewith, the most important steps of the operation are illustrated.

\section{REFERENCES}

1. Strathearn, T. C. -The problem of blindness in Palestine. Folia Ophthal. Orient., Vol. I, Fasc. 2, p. 121 ; Fasc. 3, p. $278 ; 1932-1933$.

2. Sнimкin, N. I.-Blindness among Jews in Palestine and its causes. Congress International de Medicine Tropical et d'Hygiene. Compte Rendu, Tome III, p. 825. Le Caire, 1931.

3. Tiсно, A.-Ursachen der Blindheit in Palestine. Klin. Monatsbl. f. Augenheilk., Bd. LXXVII, p. 700, 1926. 